desired human chromosome pairs. This strategy functions both to simplify mutation detection and to allow unambiguous phase information to be determined. Constructing haplotypes on the basis of conventional genotype and pedigree data is challenging, particularly for diseases with a late age of onset, such as cancer.

The homologue retained in a particular hybrid is identified by conventional genotyping of a few markers per chromosome. These "haploid" hybrids can then be used to increase the sensitivity of traditional mutation analysis, because the disease-causing chromosome will not be accompanied by the normal wild-type allele. Haplotypes may also be determined simply by genotyping each haploid hybrid. We have typed DNA from 100 hybrids to examine chromosomal retention patterns, test the feasibility of conversion for analysis of whole genomes, and evaluate assumptions regarding appropriate experimental design.

We have investigated the theoretical efficiency of using haplotypes compared with conventional genotypes in linkage and linkage disequilibrium studies. In the linkage disequilibrium setting, we determined the Fisher information (with respect to haplotype frequency) provided by unrelated individuals. For haplotypes with two single-nucleotide polymorphisms, conversion provides $5-45 \%$ more information per subject than standard genotyping, depending on true haplotype frequencies; for haplotypes with five single-nucleotide polymorphisms, improvement ranges from $20 \%$ to $92 \%$. Since Fisher information is inversely related to sample size, conversion requires up to 12.5 times fewer subjects than standard genotyping to obtain the same information. We are currently completing similar calculations in the linkage analysis setting.

The extra cost associated with conversion includes hybrid construction and characterization, and duplicate genotyping. However, the increased information per subject reduces overall recruitment and phenotyping costs, which tend to exceed those for genotyping. The efficiency of using conversion to construct haplotypes will probably increase even further as automated methods of genotyping continue to improve.

Sood, Raman

[35]

\section{Molecular cloning and expression analysis of human and mouse homologs of a new transcriptional regulator as a candidate tumor suppresor gene}

Raman Sood ${ }^{1}$, Izabela Makalowska², Pamela Pollock ${ }^{1}$, Christiane Robbins ${ }^{1}$, Suzie Chen ${ }^{3} \&$ Jeffrey Trent $^{1}$

${ }^{1}$ Cancer Genetics Branch, National Human Genome Research Institute, National Institutes of Health, Bethesda, Maryland, USA

${ }^{2}$ Genome Technology Branch, National Human Genome Research Institute, National Institutes of Health, Bethesda, Maryland, USA

${ }^{3}$ Rutgers University, Piscataway, New Jersey, USA

We report the cloning and detailed characterization of a new transcript containing two SNF2_N domains and one PHD domain. The SNF2_N domain is often seen in proteins involved in such processes as transcription regulation (for example, SNF2, MOT1), DNA repair (for example, ERCC6, RAD16), DNA recombination (for example, RAD 54), and chromatin unwinding (for example, ISWI). The PHD domain is a $\mathrm{C} 4 \mathrm{HC} 3$ zinc-finger-like motif found in nuclear proteins thought to be involved in chromatin-mediated transcriptional regulation. Shotgun sequence analysis of a mouse BAC clone identified several exons with homologies to a variety of repair protein genes in the database. Inter-exon polymerase chain reaction followed by $5^{\prime}$ rapid amplification of cloned ends and identification of expressed sequence tags for the $3^{\prime}$ end led to the cloning of a 7,225-base-pair cDNA with an open reading frame consisting of 4,848 base pairs. The human homologue was cloned by polymerase chain reaction between the expressed sequence tags identified by a BLAST search of the mouse sequence, followed by $5^{\prime}$ rapid amplification of cloned ends. The coding sequence of the two genes is $83 \%$ identical at the amino acid level. Northern blot analysis shows a transcript of approximately 7.5 kilobases in all tissues examined with both human and mouse complementary DNAs as a probe. We have mapped the human cDNA to $6 \mathrm{q} 23$, a region reported to contain a tumor suppressor locus by several laboratories, including our own. We are currently generating antibodies, and we will present preliminary functional studies.

Sorensen, Poul H.B.

[36]

\section{Ewing tumor cells activate distinct signaling pathw ays in monolayer versus anchorage-independent cultures} Elizabeth R. Lawlor ${ }^{1}$, Jerian F. Lim ${ }^{1}$, Lydia A. Taylor ${ }^{2}$,
Chris Carthy ${ }^{2} \&$ Poul H.B. Sorensen 1,2

${ }^{1}$ Departments of Pathology and Pediatrics, Children's and Women's Hospital of British Columbia, Vancouver, British Columbia, Canada

${ }_{2}^{2}$ Inphogene Biocom, Inc., Burnaby, British Columbia, Canada

The Ewing tumor (ET) family of childhood malignancies express translocationassociated EWS-ETS chimeric oncoproteins that function as aberrant transcription factors. These molecules are thought to facilitate passage through the cell cycle, contributing to increased cell proliferation. We have been comparing signaling pathways activated in ET cell lines grown in conventional monolayers with those in anchorage-independent spheroid cultures. As expected, we found that monolayer ET cells show serum-dependent activation of ERK 1/2 and p38 MAP kinases. Serum also activates PI3 kinase-dependent PKB/AKT, involved in cell survival, and induces high levels of cyclin D1/D2 protein expression. However, when the same cells are grown in spheroid cultures, there is dramatic serum-independent activation of ERK 1/2, p38, and PKB/AKT, but paradoxically cyclin D1 and D2 expression is virtually undetectable. Fluorescence-activated cell sorting analysis indicates that at least $30 \%$ of spheroid cells are cycling. This pattern of serum-independent MAP kinase activation and cyclin D1/D2 suppression can be recapitulated in monolayer cells by treatment with PI3 kinase inhibitors. To further understand changes in immediate and early gene expression that may underlie these findings, we subjected monolayer and spheroid ET cells (with and without PI3 kinase inhibitors) to gene expression profiling using Affymetrix U95A gene chips (containing over 12,000 annotated human gene sequences). The results of these experiments are currently being analyzed to identify distinct expression profiles characteristic of monolayer versus spheroid ET cells. Morphological and ultrastructural analysis of the latter indicate many similarities with primary ETs, suggesting that spheroid cultures may be a better model to study the biology of these lesions.

Sotiriou, Christos

[37]

\section{Core biopsy versus surgical tumor specimens for microarray analysis of gene expression profiles}

Christos Sotiriou $^{1}$, Khanna Chand ${ }^{2}$, David Petersen ${ }^{1}$, Amir A. Jazaeri ${ }^{1} \&$ Edison T. Liu ${ }^{1}$

\footnotetext{
${ }^{1}$ Medicine Branch, Division of Clinical Sciences, National Cancer Institute, National Institutes of Health, Gaithersburg, Maryland, USA

${ }^{2}$ Pediatric Oncology Branch, National Human Genome Research Institute, National Institutes of Health, Bethesda, Maryland, USA
}

We compare the utility of core biopsy samples with surgical tumor specimens as starting material for microarray analysis. Two human tumor xenografts, Ewing sarcoma and neuroblastoma grown in Beige-Scid mice, served as source materials for this study. Six 16g-needle core biopsies and an excision biopsy were performed on each xenograft. Three of the six core biopsies were processed separately and the 
other three were pooled together. As the initial material isolated from the core biopsies was not sufficient for microarray analysis, we performed an amplification procedure using a modified Eberwine protocol. A complementary DNA microarray consisting of 6,000 human genes was used. Comparison of the array results from core biopsies (amplified RNA) and surgical specimens (non-amplified RNA) showed maintenance of the expression profile and concordance in identifying outliers in the range of $58 \%$. This finding compares with a $48-77 \%$ concordance observed among three different samples of the same excisional biopsy using total RNA. The level of concordance was higher when an amplified core biopsy was compared with an amplified excisional biopsy: 64\% for the Ewing sarcoma and 83\% for the neuroblastoma. Pooling the core biopsies did not improve the concordance with surgical biopsies. Gene expression profiles obtained from microarray analysis differentiated Ewing sarcoma from neuroblastoma with both core and surgical biopsies as starting material. Our results suggest that core biopsy samples can be used as acceptable and reliable material for the determination of tumor genetic profiles.

Stenman, Göran K.D.

\section{Fusion of the NH2-terminal domain of the bHLH protein TCF12 to TEC in extraskeletal myxoid chondrosarcoma with translocation $t(9 ; 15)(q 22 ; q 21)$}

\author{
Helene Sjögren \& Stenman, Göran K.D. \\ Lundberg Laboratory for Cancer Research, Department of Pathology, Göteborg \\ University, Gothenburg, Sweden
}

Extraskeletal myxoid chondrosarcomas (EMC) are characterized by recurrent $\mathrm{t}(9 ; 22)$ or $\mathrm{t}(9 ; 17)$ translocations resulting in fusions of the $\mathrm{NH} 2$-terminal transactivation domains of EWS or TAF2N to the entire TEC protein. We report here an EMC with a novel translocation $t(9 ; 15)(\mathrm{q} 22 ; \mathrm{q} 21)$ and a third type of TEC containing fusion gene. The chimeric transcript encodes a protein in which the first 108 amino acids of the NH2-terminus of the basic helix-loop-helix (bHLH) protein TCF12 is linked to the entire TEC protein. The translocation separates the NH2-terminal domain of TCF12 from the bHLH domain as well as from a potential leucine zipper domain located immediately downstream of the breakpoint. These results demonstrate that the $\mathrm{NH} 2$-terminal transactivation domains of EWS or TAF2N are not essential for the oncogenic properties of fusion proteins in EMC, and that EWS or TAF2N may be replaced by a similar domain from a bHLH protein that presumably endows the fusion protein with similar functions.

Stephan, D.A

\section{Identification of expression changes of prognostic and therapeutic value in metastasizing medulloblastoma}

K.M. Brown ${ }^{1,2}$, T. MacDonald ${ }^{1}$, P.H. Cogen ${ }^{1}$, Y.-W. Chen ${ }^{1}$, K. Peterson ${ }^{1}$, Y. Chen ${ }^{3}$, B. Rood ${ }^{1}$, R.J. Packer ${ }^{1}$ \& D.A. Stephan ${ }^{1,3}$

${ }^{1}$ Childrens National Medical Center, Washington, DC, USA.

${ }^{2}$ George Washington University Genetics, Washington, DC, USA.

${ }^{3}$ National Human Genome Research Institute, National Institutes of Health,

Bethesda, Maryland, USA

Medulloblastoma is a highly malignant cerebellar tumor of children and, less frequently, of adults, with a tendency to early recurrence and dissemination. Despite recent advances in patient survival, the quality of life for survivors is poor due to neurocognitive, neuroendocrine, and hearing deficits as a direct result of the whole brain and spine radiation required for prevention of metastatic disease. Using global expression profiling, we identified genes prognostic of tumor metastasis that may also serve as new therapeutic targets. In the first phase of the study, an expression scan was done using a pooling approach, assuming that genetic heterogeneity would not be a significant confounding factor. We isolated total RNA from six metastasizing and six-non metastatic tumors, made biotinylated cRNA, and pooled samples from the two clinical groups in equimolar amounts. Gene expression profiles were generated using Affymetrix HuGeneFL Gene Chips (5600 genes). 266 genes showed differential expression greater than two-fold. The 58 genes expressed at least three-fold more or less between groups were selected for further analysis. 21/30 genes with increased expression in metastatic tumors have been implicated in promoting: invasion/metastasis (14), cell growth (4), and angiogenesis (3). 12/28 genes with decreased expression in metastatic tumors have been implicated in inhibiting: invasion/metastasis (5), cell differentiation (6), and cell growth (1). Expression of each gene is being characterized via tissue array immunostaining with $140+$ paraffin embedded tumors (45/58 genes have available antibodies). In order to evaluate the efficacy of pooling clinical samples and to identify further candidates in the second phase of the study, individual oligonucleotide arrays using $80+$ frozen tumors are being performed, and correlated with clinical and pathological data. This study has identified historically valid candidate genes involved in metastatic medulloblastoma, and has shown that a pooling strategy can be utilized effectively to identify candidate genes without complex statistical analysis, as long as validation studies are part of the study design. In addition, we show pooling may be an alternative strategy to identify relevant 'candidate' predictor genes when only small numbers of clinical samples are available.

Sulman, Erik P.

[40]

\section{Identification of candidate tumor suppressor genes in meningioma by regional expression profiling}

Erik P. Sulman ${ }^{1}$, Peter S. White 1,2 , Jan P. Dumanski³ , J ohn M. Maris ${ }^{1,2} \&$ Garrett M. Brodeur ${ }^{1,2}$

${ }^{1}$ Division of Oncology, Children's Hospital of Philadelphia, Philadelphia, Pennsylvania, USA

${ }^{2}$ Department of Pediatrics, University of Pennsylvania School of Medicine, Philadelphia, Pennsylvania, USA

${ }^{3}$ Department of Genetics and Pathology, Uppsala University, Uppsala, Sweden

Meningioma is a common and frequently recurrent central nervous system tumor. Hemizygous deletion of the short arm of chromosome 1 (1p) is second only to monosomy of chromosome 22 as the most frequently observed chromosomal alteration in these tumors. We previously identified a smallest region of overlapping deletion (SRO) spanning 1.5 centimorgans on $1 \mathrm{p} 32$; $1 \mathrm{p}$ allelic loss was predictive of tumor recurrence and correlated with loss of chromosome 22 and mutation of the NF2 tumor suppressor gene. Deletion of this region has also been observed in oligodendroglioma and neuroblastoma. To identify transcripts in the SRO, we constructed a physical map of the region. We have collected 80 largeinsert clones assembled into 4 contigs spanning approximately 3 megabases. Preliminary analysis of clone sequence has identified several previously unmapped genes in the SRO. A total of 78 unique transcripts have been localized to the region. We identified additional genes of interest through analysis of the expression differences between a normal meninges cell line, LTAg2B, and a meningioma cell line, KT21MG1, using Atlas Cancer Gene blots. To identify candidate tumor suppressor genes we are profiling the expression of all identified transcripts as well as other genes previously proposed to play a role in meningioma tumorigenesis, such as NF2, LIF and ADTB1, using a custom-made, low-resolution, glass slide complementary DNA microarray. Ultimately we plan to obtain a panel of candidate transcripts exhibiting tumor-specific changes in expression that can be examined further for genomic rearrangements or for mutations in tumors with characteristic deletions in the SRO. 